\title{
Enseñanza de la geometría en la escuela primaria
}

\author{
Cómo entrelaza el maestro, en sus prácticas, \\ la matemática, el contexto y sus alumnos $(*)$
}

Ariel Fripp **

\section{Resumen}

En este trabajo se presentan las líneas centrales que articularon una investigación acerca de la construcción metodológica de las prácticas de enseñanza de la Geometría en la escuela primaria.

Esa investigación se sustentó en una concepción positiva del trabajo de los docentes del nivel primario, tomando como hipótesis la convicción de que ellos reflexionan sobre sus prácticas; interesó buscar datos que iluminaran las maneras singulares en las que esos docentes construyen las prácticas de aula en el momento de enseñar Geometría intentando responder si esa construcción difiere o no según el objeto de estudio y el área de conocimiento al cual pertenece. Para ello, a través de entrevistas en cuatro colegios privados de la ciudad de Montevideo se pudo llegar a categorizar las prácticas de enseñanza geométrica, como prácticas de materialización, de utilidad, de problematización, de reiteración, de sobrevuelo y de responsabilidad social.

PALABRAS CLAVE: Enseñanza, Aprendizaje, Geometría, Investigación Cualitativa.

\section{Abstract}

The present paper introduces the main lines articulating an investigation on the construction methodology of teaching practices in elementary school Geometry. The research was sustained by a positive conception of the work of elementary school teachers, taking as hypothesis the conviction that educators reflect on their practices. Of particular interest was to obtain data that would shed light on the particular ways each teacher constructs classroom practices while teaching Geometry, and attempt to respond whether this construction differs or not, depending on the object of study and the area of knowledge it belongs to. To achieve these objectives, several interviews with four private schools in the city of Montevideo were performed, and it was possible to classify teaching practices in Geometry into five categories: practices of utility, problematizing practices, practices of repetition, overflight practices and social responsibility.

KEY WORDS: Teaching, Learning, Geometry, Qualitative Research.

\section{Introducción}

En esta investigación realizada en el período 2009-2010, se consideró oportuno situarse en un lugar un poco diferente a los usuales, desde el cual estudiar las actividades de enseñanza de los maestros uruguayos. Es común observar trabajos de formación inicial o en servicio dirigidos a maestros de enseñanza primaria basados en lo que al docente le falta o en lo que no sabe; suelen planificarse las acciones de formación o de investigación desde lo que el maestro no tiene. "La lectura en negativo reifica las relaciones para hacerlas cosas, nihiliza esas cosas transformándolas en cosas ausentes, 'explica' el mundo por desplazamiento de las carencias, postula una causalidad de la carencia. Este tipo de lectura engendra 'objetos'”. (Charlot, 2006, 36). Remitirse solamente a marcar lo que falta, a señalar lo que está mal parecería resguardar a quien lo hace en una cierta neutralidad ajena a lo que efectivamente ocurre. El profesional que 
lee en negativo las prácticas de enseñanza de otro, critica y de esta manera se autoprotege, es parte de una lógica de observación del tipo pretzel (Kincheloe, 2001) donde la lectura en negativo de las enseñanzas en Geometría sólo genera prácticas negativas.

La investigación realizada, en el marco de la Maestría de Educación de la Universidad ORT, se centró en leer las maneras mediante las cuales un maestro de educación primaria llega a tomar decisiones sobre sus acciones de enseñanza de la Geometría. Importó investigar este aspecto y recuperar al maestro como generador de buenas enseñanzas, reconociendo así a la práctica como una de las posibles fuentes de conocimiento didáctico. Leer positivamente las prácticas de enseñanza de la Geometría implicó buscar buenas enseñanzas, lo que conllevó buscar las fuertes razones morales y epistemológicas que las sustentan. Analizarlas positivamente, en sus condiciones naturales y en los contextos donde se desplegaban exigió también, parafraseando a Jackson (2002), centrar la atención no tanto en lo que se vio como en lo que se leyó.

Se partió de la premisa de que la Geometría, como área del conocimiento matemático es una construcción del hombre, y sus características son comunes y conocidas por la comunidad; en cambio la enseñanza de la Geometría no es común a los distintos colectivos de enseñantes. Fue oportuno preguntarse, ¿cómo se enseña Geometría en los diferentes contextos socioculturales?, ¿qué cuestiones son tenidas en cuenta en el momento de planificar una clase de Geometría?, ¿son las mismas cuestiones que se atiende cuando se enseña un concepto perteneciente a otra área del conocimiento?

Se basó el estudio en la definición de construcción metodológica; Jaramillo $(2008,11)$ plantea que las construcciones metodológicas son "las maneras específicas o los estilos particulares construidos por los docentes en sus prácticas de enseñanza, para propiciar la adquisición de conocimiento y los procesos de formación de los estudiantes." Se abordó y atendió a este concepto en un abordaje metodológico que se cargó de sentido al ser considerado como problema de conocimiento didáctico que no podía desconocer el objeto geométrico. Para considerar las prácticas de enseñanza de la Geometría como construcciones metodológicas no se pudo obviar la relación existente entre la estructura de la Geometría y la estructura cognitiva del alumno escolar, que aprende en un contexto determinado. La singularidad del tejido de esos aspectos en la arquitectura con la que cada docente proyectaba y desarrollaba sus prácticas áulicas fue el centro de atención. Dentro de esa singularidad se indagó acerca del lugar que los maestros otorgaban al niño/alumno en la construcción -génesis y cambio- de la cultura académica geométrica; cultura en el sentido de negociación constante de significados (Bruner, 1988). Se atendió al maestro como portador de cultura geométrica y al alumno como sujeto activo de aprendizaje que discrepa, tensiona y genera constantes reestructuras en el proceso de aculturación. Se pensó en la posible existencia de una relación tensa y creativa entre el maestro y los alumnos, tensión que podía generar construcciones culturales geométricas singulares y novedosas. Al reconocer a la Geometría como creación cultural del hombre, se consideró tanto al maestro como al niño como activos participantes en el acto de crearla y recrearla.

En síntesis se compartió el planteo de Gloria Edelstein (2002) quien establece que un docente que atiende, en la medida de lo posible, la epistemología de un área del conocimiento (en este caso de la Matemática y en especial de la Geometría), las peculiaridades de sus alumnos y por lo tanto de sus aprendizajes, y se reconoce como parte de una cultura escolar determinada genera un estilo particular de enseñante: "(...) se entiende que la tarea del profesor en el aula consiste,(...) en elaborar un modo personal de intervención que se concretiza en opciones diversas acordes a la situación en que le corresponde actuar." (Edelstein, 2002, 473) 


\section{Metodología}

El estudio sobre la construcción metodológica de las enseñanzas de la Geometría en la escuela primaria, se resguardó en un marco investigativo de tipo cualitativo. Interesó al igual que Tesch, citada por Tojar $(2006,143)$ atender "un enfoque de producción de conocimientos." Esta producción de conocimiento deseó ocuparse del maestro desde la perspectiva del propio maestro respetando y atendiendo el contexto donde desarrolla su labor. La etimología de la palabra contexto, nos remite a aquello que 'está entrelazado', por lo que se hizo imprescindible elegir métodos y técnicas que respetaran dicho entrelazado pero que a su vez permitieran ver entre sus hebras. Se investigó desde una perspectiva fenomenológica, por lo que se consideró esencial "centrarse en las experiencias e interpretaciones de los fenómenos, por parte de la gente que los vive" (Valles, 1999, 64-65).

Concebir las construcciones metodológicas de las prácticas de enseñanza como objeto de investigación y estudiarlas bajo el paradigma cualitativo desde el aporte fenomenológico implicó decidir cuáles serían las técnicas de investigación a utilizar, qué lugar y qué función se le adjudicarían a ellas y de qué manera se analizarían los criterios de verdad. No se persiguieron criterios de verdad tradicionales como la validez interna y externa, confiabilidad y objetividad por considerar que ello entraría en contradicción con la investigación que se desarrollaba. Por el contrario pareció oportuno buscar otros criterios (Contreras, 1994) como lo son la credibilidad, la posibilidad de transferencia, la fidelidad y la posibilidad de confirmación. Se buscó también la mirada de un especialista en educación matemática como herramienta de validación por valorar en él su profundo conocimiento de la Didáctica de la Matemática.

\section{2.a. Técnicas}

Se consideró oportuno sembrar y recolectar datos utilizando entrevistas. Las entrevistas, herramientas de investigación, fueron tenidas en cuenta como "extensiones del instrumento humano, ayudas a la memoria y visión." (Arnal et al. 1999, 202). El propio investigador fue uno de los instrumentos esenciales en el momento de indagar.

El trabajo de campo ocurrió atendiendo a tres momentos que condicionaron el tipo de entrevistas que sucedieron en cada uno de ellos. El siguiente cuadro da cuenta de esos momentos.

\section{MOMENTOS DEL TRABAJO DE CAMPO}

1. Entrevistas a directivos.

2. Entrevistas a maestros.

3. Entrevista a un actor clave. 


\section{2.a.a. Entrevistas informales a directivos}

Se entrevistó a cuatro integrantes de equipos directivos de colegios privados de la ciudad de Montevideo (directores o coordinadores pedagógicos), considerados ellos como informantes calificados que identificaron en sus instituciones a aquellos maestros que generaban, a su entender, buenas prácticas de enseñanza de la Geometría. Iniciar el muestreo teórico con directivos con cargo de directores o coordinadores pedagógicos se constituyó en una decisión de tipo estratégica pues facilitó la accesibilidad a cada uno de los colegios. Se consideró importante que las instituciones elegidas se diferenciaran en la población estudiantil que atendían. En ese sentido, y para dar cuenta de una necesaria variación, se tuvo en cuenta el contexto de cada colegio como dimensión teórica. Inicialmente se seleccionaron cinco colegios, finalmente se trabajó en cuatro de ellos; dos colegios de contexto muy favorecido y dos de contextos desfavorecidos. Se buscó variación, pero no se optó por una variación extrema donde ingenuamente se intentara representar todas las categorías de colegios privados de la ciudad de Montevideo. La elección de estos colegios fue decidida también por el conocimiento que se tenía del perfil de sus directivos y su acercamiento a la enseñanza de la Matemática. Se realizaron entonces cuatro entrevistas, de tipo informal, a maestros con cargos supervisores; se trabajó con una directora, dos subdirectoras y una coordinadora pedagógica. La coordinadora pedagógica es una maestra con una muy amplia y fructífera carrera docente dentro de educación primaria; ha sido directora en escuelas públicas y privadas de la ciudad de Montevideo. La directora entrevistada posee una larga carrera en la enseñanza primaria pública y se caracteriza por una constante búsqueda en su formación matemática, evidenciada en los numerosos cursos de formación en servicio que tiene aprobados. Las dos subdirectoras han participado de instancias de especialización como líderes pedagógicas de instituciones educativas con profundización en Lenguaje, Ciencias y Matemática.

\section{2.a.b. Entrevistas abiertas a maestros}

Se efectuaron entrevistas abiertas habilitando a los maestros a narrar aquellas experiencias que ellos valoraban como sus mejores clases de Geometría, invitándolos a desarrollar y justificar sus puntos de vista. Se entrevistó a aquellos maestros que los directivos consideraron buenos en cuanto a sus prácticas de enseñanza de la Matemática. Inicialmente se respetó la lista inicial (diez maestros) confeccionada con los aportes de cada director pero, como plantean Taylor y Bogdan (1987) no importó tanto la cantidad de entrevistados sino su potencial informativo. Se entrevistaron ocho maestros ya que los datos que se iban construyendo no producían comprensión nueva; se produjo saturación de la información.

\section{2.a.c. Entrevista al actor calificado}

La entrevista a esta personalidad relevante en el área de la formación matemática de maestros, se estructuró como consecuencia de los datos recogidos en las entrevistas a los maestros de aula; estos datos se constituyeron en sustentos de una guía que convirtió a esta entrevista en una entrevista dirigida. Se le proporcionaron fragmentos con apreciaciones de los maestros investigados solicitándole su opinión; según las respuestas que se iban obteniendo se le entregaba otro tramo de entrevista transcripta que permitía ahondar en lo planteado o que contrastaba con su opinión. Este diseño no se consideró como perteneciente a la fase de entrada, al decir de Morse (1994) citada por Valles (1999), sino que fue un momento del trabajo que se solapó con la fase de recogida productiva y análisis. 


\section{Hallazgos}

\section{3.a. Categorización de las prácticas de enseñanza de la Geometría}

"Todo objeto o práctica es significada de alguna manera al ser apropiada por los agentes sociales. Toda configuración social es discursiva en este sentido. Las prácticas educativas, en tanto prácticas sociales, son también discursivas." (Buenfil, 1993, 5)

Al enfrentarse, como investigador, al cúmulo de información que las entrevistas a los docentes brindaron, se tuvo la necesidad de identificar esencialidades. Así como Picasso en su obra La metamorfosis del toro. Estilización y síntesis partió de imágenes taurinas cargadas de detalles para quedarse con lo esencial, es decir con aquello que era común a todos los toros y que a su vez los identificaba como animales singulares, en la investigación desarrollada se pudieron construir categorías esenciales que hacen a las prácticas de enseñanza de la Geometría de los maestros sujetos de esta investigación.

\section{PRÁCTICAS DE ENSEÑANZA DE LA GEOMETRÍA}

\author{
De Materialización y de Utilidad \\ De Responsabilidad Social \\ De Problematización \\ De Sobrevuelo y de Reiteración
}

\section{3.b. Prácticas de materialización y de utilidad}

Se encontró que los maestros explicitaban como característica esencial de la Geometría, un alto nivel de abstracción. Pudo establecerse que esta concepción generaba dos tipos de acciones por parte de ellos, a las que se llamó Prácticas de Materialización y Prácticas de Utilidad. Mediante las Prácticas de Materialización, los maestros, intentan que los alumnos posean o construyan referentes materiales o físicos de conceptos geométricos. Es oportuno destacar que las cuestiones que los maestros materializaban se correspondían a figuras geométricas del plano y del espacio; en ningún momento hicieron referencia a materializaciones de relaciones geométricas. Cuando se trianguló esta observación, apelando a la opinión del experto, éste coincidió en que las maestras efectuaban asociaciones muy directas con las representaciones de los objetos.

Por otro lado, mediante las Prácticas de Utilidad los docentes intentaban sortear la dificultad que detectaban en la abstracción de los conceptos geométricos; remitían los trabajos de aula a la posible utilización de los contenidos abordados y con bastante frecuencia interrogaban a sus alumnos y se cuestionaban sobre la utilidad de lo que enseñaban. Los maestros, en las Prácticas de Utilidad, enfatizaban la búsqueda y explicitación de utilidad y aplicación de los conceptos geométricos pero no daban cuenta de su importancia como construcción intelectual.

\section{3.c. Prácticas de responsabilidad social}

Esas prácticas forman parte de aquellas acciones llevadas a cabo por el maestro que intentan responder a lo que ellos consideran parte esencial de su tarea como educadores. En cierta manera a través de estas prácticas los docentes intentaban cumplir con aquellas cuestiones que creen que la sociedad esperaba de ellos, cuestiones referidas más a la adquisición de hábitos de tipo social que a la adquisición y construcción de conocimientos geométricos. Se realizaron otras miradas a las entrevistas, enriquecidas con los aportes de especialistas en análisis de discursos o contenidos. Basándose en trabajos de Bardin (1986) y Van Dijk (2003) pudo detectarse información implícita en los textos obtenidos al desgrabar las entrevistas; los docentes daban cuenta que no podían, ni debían dejar de enseñar Geometría aunque no fuera el área en la cual se sentían más seguros. Sentían también como responsabilidad social 
colaborar a que todos sus alumnos terminaran la tarea geométrica planteada; parecía primar la generación de un hábito de tipo social por sobre el aprendizaje matemático.

\section{3.d. Prácticas de problematización}

En contraposición a las ya mencionadas Prácticas de Materialización o de Utilidad, se encontraron otras basadas en la problematización de los conceptos geométricos. Con ellas los maestros ubicaban en un lugar preponderante a las relaciones que existen hacia el interior de las figuras y entre las figuras geométricas mismas. El interés y por lo tanto el origen en la construcción de las Prácticas de Problematización radicaba en los propios objetos geométricos y las relaciones que se dan en ellos y entre ellos; con estas actividades los maestros reconocían que incentivaban a sus alumnos en el desarrollo del pensamiento geométrico. Las Prácticas de Problematización fueron consideradas, por quien realizó esta investigación, como Prácticas de Buena Enseñanza -Fenstermacher (1989); Litwin (2008)- ya que daban cuenta de un maestro cargado de buenas intenciones, que intentaba respetar epistemológicamente el objeto geométrico y que además reconocía al niño como sujeto de aprendizaje.

\section{3.e. Prácticas de sobrevuelo y de reiteración}

En el transcurso del estudio de los datos surgió una interrogante referida a la posible afectación que el contexto podía provocar en la generación de actividades de enseñanza geométrica. Al analizar los discursos de los docentes entrevistados se pudieron reconocer elementos comunes entre algunas de sus prácticas. Se comparte el planteo que hacía una de las maestras entrevistadas pues se considera que él ejemplifica claramente el origen de una de las categorizaciones que nos ocupa en este momento:

"Entonces tú das los conceptos, los das a la apurada, los das sobrevolando porque estás trabajando con un concepto y lo tenés que suspender porque vas a atender a aquel que se estaba peleando, que estaba mirando para afuera... volvés al concepto... volvés a suspender porque tenés que estar con este otro que se le cayó el cuaderno, que no tiene juego de geometría."

Si sobrevolar significa volar sobre, el término empleado en la cita anterior da cuenta de una enseñanza que ocurriría por fuera o por arriba del grupo de alumnos. Al relacionar 'volar sobre' con la palabra 'das', se pudo inferir una postura de poco compromiso, al menos intelectual, con el alumno de contexto desfavorecido. Más que considerar al niño como sujeto de aprendizaje parecería que se lo consideraba como depositario de algo que se le debe dar cuando se vuela sobre él. En las Prácticas de Sobrevuelo anida la concepción de Geometría como algo externo al alumno y aprender implicaría poder recibir lo que el maestro le brinda. En estas prácticas de enseñanza, el docente es el que sabe y el alumno es el sujeto al que se le informa parte de ese saber.

Los entrevistados también explicitaron otra característica cuando narraron sus clases de enseñanza de la Geometría, reconocieron como acción común en sus aulas un continuo ir y venir en lo que se está enseñando. Esa práctica, que bien podría llamarse de Reiteración o de ir y venir, no era una simple e ineficaz repetición sino una visita al concepto geométrico desde situaciones diferentes que aportaban otros significados y favorecían que el sentido y razonamiento geométrico del niño se ampliara.

\section{3.f. ¿El contexto conmueve al método?}

Una postura sostenida a lo largo de toda la investigación consistió en destacar que una práctica de enseñanza adquiere real importancia cuando se reconoce atravesada por el contexto en el cual se desarrolla. En un nivel básico o primario, se pudo detectar que los maestros 
investigados contemplaban la conformación de la clase en cuanto a cantidad de niños y a dinámicas de trabajo empleadas, por lo que se pudo afirmar que atendían entonces a uno de los componentes del contexto: la constitución del grupo de clase. En un segundo nivel se indagó en la atención o no, por parte de los maestros, del capital sociocultural y afectivo de los alumnos al momento de construir sus enseñanzas geométricas. Se buscaron relaciones o implicancias del contexto de los niños con las metodologías empleadas por los docentes

\section{3.g. El tiempo}

Fueron de dos tipos los hallazgos que relacionaban la característica sociocultural y afectiva del colegio en el cual se trabajaba y la labor del docente. En aquellas instituciones más desfavorecidas se constató que el factor que los maestros mencionaban en forma reiterada era el tiempo. Pudo concluirse que aun detectando a este factor como siempre presente en el discurso de ellos, el mismo no generaba una nueva y singular forma de hacer y enseñar Geometría. El contexto desfavorable no conmovió al método.

\section{3.h. La supervisión parental}

Los docentes, cuyos alumnos pertenecen a contextos socioculturales más favorecidos, plantearon inicialmente algunas diferencias en su trabajo, al comparar experiencias personales en escuelas públicas de contextos críticos. Esas diferencias se centraban en la posesión o no de condiciones materiales más ricas en las instituciones privadas y en un marcado oficio de alumno en los niños de estas instituciones. Al profundizar en el análisis pudo detectarse que los investigados reconocían que el contexto, a través de los padres de sus alumnos, afectaba sus prácticas de enseñanza de la Geometría. Se encontró que ciertas tareas que en otro momento eran enviadas para ser resueltas en el domicilio, ya no se remitían con la intención de minimizar la interferencia paternal. Los maestros reconocieron que los problemas geométricos enviados como tareas domiciliarias estaban ahora sujetos a un mayor análisis por parte de ellos con la intención de que los padres no lo hicieran, o si los hacían, pudieran entenderlos. Se podría plantear entonces que en los colegios más favorecidos, el contexto sí conmovió al método a través de la llamada Supervisión Parental.

\section{3.i. La búsqueda de pautas ¿qué conectan?}

"La visión correcta del mundo es aquella que atiende a las relaciones dinámicas" Gregory Bateson (1993, 390)

Los maestros entrevistados reconocieron que ellos no eran el mismo docente en el momento de enseñar Geometría o al momento de trabajar con otro eje temático. Esta percepción se basaba en cierta inseguridad relativa a las características de su formación en Geometría, lo que imprimía una debilidad en su biografía profesional. Más allá de esta diferencia pudo encontrarse en sus planteos, gracias a los aportes de Van Dijk (2003), ciertas palabras que formaban parte de un discurso muy general con ribetes pedagógicos, o en algunos casos vinculados a cuestiones pertenecientes a una concepción didáctica que no atendía la especificidad del objeto geométrico. Hacían referencia a planificaciones o intenciones comunes a la enseñanza de diferentes objetos de conocimiento, sin atención a la especificidad de cada uno de ellos; parecía que los entrevistados eran portadores de ciertas reglas de enseñanza aplicables a cualquier situación y a cualquier concepto a enseñar.

\section{Conclusiones}

Se considera que la importancia del tema investigado radicó en la posibilidad de detectar cuáles eran las cuestiones de las cuales se valían los docentes de educación primaria, sujetos de esta investigación, para enseñar los conceptos geométricos y cuáles eran las formas que tenían para hacerlo. 


\section{4.a. La abstracción}

En primer lugar se destaca la concepción de Geometría que fue compartida por el colectivo docente; para ellos la característica fundamental de la Geometría es su alto grado de abstracción y desde esa concepción construían sus prácticas de enseñanza. Se arribó a la conclusión que para ellos el objeto geométrico a enseñar tiene un grado de abstracción mayor que cualquier otro objeto matemático de enseñanza y parecería que si se lo comparara con el perteneciente a otra área del conocimiento, sucedería lo mismo.

En ningún momento pudieron explicitar la condición abstracta que tiene todo objeto de conocimiento.

La abstracción de la Geometría se halló como sustento -con diferente fuerza- en cuatro de las seis categorizaciones de las prácticas que fueron construidas: en las Prácticas de Materialización, de Utilidad, de Problematización, de Reiteración y de Sobrevuelo.

\section{4.b. La construcción metodológica de las prácticas de problematización como buena práctica de enseñanza}

Otro aspecto a destacar, surgido de la construcción de la categoría llamada Prácticas de Problematización se refiere a la relación hallada entre el paradigma de las Buenas Prácticas de Enseñanza -Fenstermacher (1989); Litwin (2008)- y la Construcción Metodológica -Edelstein (1996). Pudo inferirse que los maestros, a través de la construcción metodológica de sus actividades dieron cuenta de buenas prácticas de enseñanza. Algunos maestros investigados construyeron metodológicamente buenas prácticas de enseñanza de la Geometría a través de las llamadas Prácticas de Problematización.

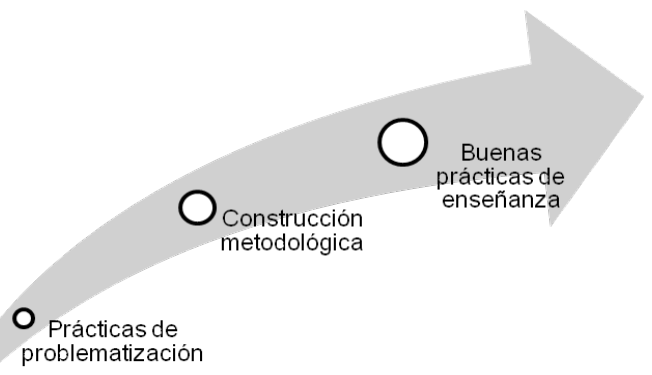

\section{4.c. El contexto}

Al análisis de los datos que la empiria brindó, permitió también afirmar que el contexto no conmovía medularmente al método de enseñanza, no generó construcciones singulares de las prácticas de enseñanza, no promovió un tratamiento del objeto geométrico que atendiera las particularidades del ámbito en el cual se enseñaba. La única implicancia que se pudo encontrar tuvo que ver con la presencia de los padres y su influencia en algunas cuestiones referidas a la enseñanza de la Geometría; ese influjo, pseudoepistemológico, fue categorizado y nominado como Supervisión Parental.

\section{4.d. La enseñanza de la Geometría y la enseñanza de otras áreas}

¿Y qué ocurrió con la enseñanza de la Geometría y la enseñanza de otras áreas del conocimiento? Se pudieron construir datos que respondieron a esa interrogante y permitieron concluir que las maestras participantes de esta investigación, poseían una postura pedagógica y didáctica que parecería no reconocer especificidad de los distintos objetos de conocimiento. 


\section{Bibliografía}

Arnal, Justo et al. 1999. Investigación educativa. Fundamentos y metodologías. Barcelona: Editorial Labor, S.A.

Bardin, L. 1986. Análisis de contenido. Madrid: Akal ediciones.

Bateson, G. 1993. Una unidad sagrada. Pasos ulteriores hacia una ecología de la mente. Barcelona: Gedisa.

Bruner, J. 1988. Realidad mental y mundos posibles. Los actos de la imaginación que dan sentido a la experiencia. Barcelona: Gedisa.

Buenfil, Rosa Nidia. 1993. Análisis de discurso y educación. Cuaderno DIE, 26. Consultado en diciembre de 2011 en http://www.die.cinvestav.mx/Portals/0/SiteDocs/Investigadores/ RBuenfil/Articulos/AnalisisDeDiscursoYEducacion.pdf

Charlot, B. 2006. La relación con el saber. Elementos para una teoría. Montevideo: Trilce. Contreras Montes de Oca, I. 1994. Revista de la Universidad de Costa Rica. Vol.18. N².

Edelstein, G. 1996. El método en el debate didáctico contemporáneo. En Alicia Camilloni et al. 1996. Corrientes didácticas contemporáneas. Buenos Aires: Paidós.

Edelstein, G. 2002. Problematizar las prácticas de la enseñanza. En Revista Perspectiva, Vol. 20. №2. 467-482. Florianópolis.

Fenstermacher, G. 1989. Tres aspectos de la filosofía de la investigación sobre la enseñanza. En Wittrock, M.: La investigación en enseñanza, tomo 1. Madrid: Paidós.

Jackson, P. 2002. Práctica de la enseñanza. Buenos Aires: Amorrortu.

Jaramillo, J; GAITÁN, C. 2008. Caracterización de prácticas de enseñanza universitaria. Revista Educación y Desarrollo Social. Vol.2. №2. Bogotá.

Kincheloe, J. L. 2001. Hacia una revisión crítica del pensamiento docente. Barcelona: Octaedro.

Litwin, E. 2008. El oficio de enseñar. Condiciones y contextos. Buenos Aires: Paidós.

Taylor, S.J.; Bogdan, R. 1987. Introducción a los métodos cualitativos de investigación. Barcelona: Paidós Básica.

Tojar Hurtado, J. C. 2006. Investigación Cualitativa. Comprender y actuar. Madrid: Editorial La Muralla.

Valles, M. S. 1999. Técnicas cualitativas de investigación social. Reflexión metodológica y práctica profesional. Madrid: Editorial Síntesis, S.A.

Van dijk, Teun A. 2003. La multidisciplinaridad del análisis crítico del discurso: un alegato a favor de la diversidad. En Wodak, R. y Meyer, M. 2003. Métodos de análisis crítico del discurso. Barcelona: Gedisa.

\footnotetext{
* Fecha de recibido: 6/02/2012

Fecha de aceptado: 30/03/2012
}

\footnotetext{
** Master en Educación de la Universidad ORT Uruguay. Profesor de Matemática, Instituto de Profesores Artigas. Autor de varios libros de Matemática y Didáctica de la Matemática y de artículos referidos a la enseñanza de estas disciplinas. Coordinador de Gestión de Proyectos Centrales, Consejo Directivo Central (CODICEN) e Inspector de Matemática del Consejo de Educación Secundaria (CES). Tutor e Investigador, Instituto de Educación, Universidad ORT Uruguay.
} 